\title{
TUBERCULOSIS
}

\section{Pigtail drainage in the treatment of tuberculous pleural effusions: a randomised study}

\author{
Y-F Lai, T-Y Chao, Y-H Wang, A-S Lin
}

Thorax 2003;58:149-152

Background: Tuberculous pleurisy can result in obvious clinical symptoms, pleural fibrosis, and pleural thickening. Some studies of tuberculous pleurisy have suggested that symptomatic improvement and minimisation of sequelae can be achieved by completely draining the effusion during treatment, although the results have not been conclusive.

See end of article for authors' affiliations

Methods: Sixty one patients with tuberculous pleurisy were divided into two groups; 30 patients received pigtail drainage combined with antituberculosis (TB) drug treatment and 31 received only anti-TB drugs. Outcome measurements were assessed for a period of 24 weeks after treatment and included symptom scores and the incidence of residual pleural thickening (RPT).

Correspondence to: Dr Y-F Lai, Division of Pulmonary Medicine, Department of Internal Medicine, Chang Gung Memorial Hospital, 123, Ta Pei Road, Niao Sung Hsiang, Kaohsiung Hsien, Taiwan;

young@adm.cgmh.org.tw

Revised version received 2 September 2002 Accepted for publication 2 October 2002 Results: Although the duration of dyspnoea was significantly shortened by the use of pigtail drainage (median 4 days (IQR 4-5) v 8 days (IQR 7-16), p<0.001), a comparison of combined mean (SD) visual analogue scale (VAS) scores showed no significant difference between the groups after one week of treatment (57.1 (33.2) v 68.5 (44.7) or at any time during the follow up period. The incidence of RPT of more than $10 \mathrm{~mm}$ in the group treated with pigtail drainage and anti-TB drugs was $26 \% \mathrm{com}$ pared with $28 \%$ in the group receiving drug treatment only. The incidence of RPT levels of more than $2 \mathrm{~mm}$ in the two groups was $50 \%$ and $51 \%$, respectively. No statistical difference between the two groups in terms of forced vital capacity was found at the end of treatment (median (IQR) 85.5\% (6994) of predicted $v 88 \%$ (78-96) of predicted).

Conclusion: The addition of pigtail drainage to an effective anti-TB regimen is not clinically relevant and does not reduce the level of RPT.

W hile new therapeutic regimens can control tuberculous pleural effusions, residual pleural thickening (RPT) has been found in about half the patients treated in a number of studies. ${ }^{1-3}$ To help reduce RPT and facilitate recovery, therapeutic thoracentesis or early complete drainage in addition to anti-tuberculosis (TB) drugs has been tried to obtain symptomatic improvement. However, the results have not been conclusive ${ }^{245}$ so we have conducted a randomised study to investigate the possible benefit of pigtail drainage in these patients. To our knowledge, no other controlled studies of this form of treatment have been published.

\section{METHODS}

Between December 1998 and June 2000 all patients older than 17 years with an onset of pleural effusion not treated elsewhere were admitted for further evaluation. All patients received a needle pleural biopsy and diagnostic thoracentesis (less than $50 \mathrm{ml}$ ) on the first day of hospitalisation. Tuberculous pleurisy was confirmed in each patient by the presence of caseating granulomas, either with or without acid fast bacilli on histological examination. Other causes of granulomatous disease were excluded. Informed signed consent was obtained from all patients eligible to participate in the study.

Participating patients were randomised to one of two treatment regimens: standard anti-TB drug treatment combined with pigtail drainage or standard anti-TB drug treatment only. The standard anti-TB treatment included isoniazid $(300 \mathrm{mg} /$ day), rifampin ( $450 \mathrm{mg} /$ day in patients $<50 \mathrm{~kg}$ body weight; $600 \mathrm{mg} /$ day in patients $\geqslant 50 \mathrm{~kg}$ body weight), ethambutol $(800 \mathrm{mg} /$ day), and pyrazinamide $(1500 \mathrm{mg} /$ day $)$. With the exception of pyrazinamide which was administered during the initial 2 months only, all anti-TB drugs were administered for a total of 6 months.
Standard posterior-anterior and lateral chest radiographs were taken of each patient on admission and on the fourth and seventh days following admission. Chest radiographs were taken on a monthly basis during outpatient follow up visits up to 1 month after completion of the full treatment course. At initial presentation the amount of pleural effusion was recorded as small (less than one third of one hemithorax), moderate (between one third and two thirds of one hemithorax), or large (more than two thirds of one hemithorax). The test further defined two degrees of pleural thickening-first RPT (pleural thickening in excess of $10 \mathrm{~mm}$ at maximum thickness point); second RPT (pleural thickening of 2-10 mm) - using the presence or absence of RPT.

Standard spirometric tests were performed during the first, fourth, and sixth months using a Vitalograph Spirotrac III (Vitalograph Inc, USA). Pulmonary function measurements were performed in accordance with American Thoracic Society guidelines. ${ }^{6}$ A visual analogue scale (VAS) was used to grade dyspnoea, cough, night sweating, fatigue, appetite, pleuritic chest pain, and general well being on a scale of $0-100{ }^{7}$ The effects of pigtail drainage were evaluated by: (1) the number of days fever and dyspnoea persisted after treatment initiation; (2) the degree of improvement in VAS score; (3) forced vital capacity (FVC) at the end of the treatment period; and (4) RPT 6 months after treatment.

\section{Statistical analysis}

Numerical data were presented as mean (SD) values. Median values with interquartile range (IQR; $25 \%$ and $75 \%$ ) were calculated for the skewed data. Repeated measures analysis of variance was used to test the difference in VAS scores between subjects (within a group in weeks and between pigtail drainage). For between group comparisons the Mann-Whitney U test was used for two sample comparison of non-normally distributed numerical data. $\chi^{2}$ tests were performed for two 
Table 1 Characteristics of the study population

\begin{tabular}{|c|c|c|}
\hline & $\begin{array}{l}\text { Drainage group } \\
(n=30)\end{array}$ & $\begin{array}{l}\text { No drainage } \\
\text { group }(n=31)\end{array}$ \\
\hline$M / F$ & $19 / 11$ & $18 / 13$ \\
\hline Mean (SD) age (years) & $61.5(17.7)$ & $56.6(22.4)$ \\
\hline $\begin{array}{l}\text { Median time from onset of } \\
\text { symptoms to treatment days (IQR) }\end{array}$ & $11(9-30)$ & $8(7-14)$ \\
\hline Patients with risk factors* & $11(36 \%)$ & $7(23 \%)$ \\
\hline $\begin{array}{l}\text { Pleuritis combined with pulmonary } \\
\text { tuberculosis (\%) }\end{array}$ & $9(30 \%)$ & $11(35 \%)$ \\
\hline \multicolumn{3}{|l|}{ Initial amount of pleural effusion } \\
\hline Small & 2 & 2 \\
\hline Moderate & 15 & 16 \\
\hline Large & 13 & 13 \\
\hline \multicolumn{3}{|c|}{$\begin{array}{l}\text { IQR=interquartile range. } \\
\text { *Risk factors including oral steroid, diabetes mellitus, liver cirrhosis, } \\
\text { subtotal gastrectomy and alcoholism. }\end{array}$} \\
\hline
\end{tabular}

sample comparison of proportions. p values of $<0.05$ were considered statistically significant. Statistical analyses were performed using commercially available software (SPSS Version 10.0; SPSS Inc, Chicago, Illinois, USA and Stata Statistical Software; Release 7.0, College Station, TX, USA).

\section{RESULTS}

Sixty six patients were diagnosed with tuberculous pleurisy; five were excluded from the final analysis because of non-compliance with treatment requirements and loss to follow up (three in pigtail drainage group and two in control group). Of the 61 remaining patients, 30 received pigtail drainage in combination with standard anti-TB treatment and 31 received only the standard anti-TB treatment. The characteristics of these patients are shown in table 1. There were no significant differences between the two groups.

Table 2 shows the duration of fever and dyspnoea, pleural changes, and the FVC at the completion of treatment. Median (IQR) time for fever subsidence was 0 days (0-6) for those receiving both anti-TB drugs and pigtail treatment and 0 days $(0-5)$ for those receiving anti-TB treatment only. The difference between the two groups was not significant. Dyspnoea improved more rapidly in the dual treatment group than in the single treatment group (median (IQR) 4 days $(4-5) \quad v 8$ days $(7-16) ; \mathrm{p}<0.001)$. Improvement in the individual VAS scores and combined VAS index scores was significantly different after 1 week of treatment in both groups. However, the difference between the two groups was not statistically significant at any other follow up evaluation (table 3). On completion of treatment no significant difference was seen between the two groups in the incidence of RPT, either $>2 \mathrm{~mm}$ or $>10 \mathrm{~mm}$. Lung function was also similar in the two groups following completion of treatment (median (IQR) $85.5 \%$ (69-94) in the dual treatment group and $88 \%$ (78-96) in the single treatment group.

After a 6 month postoperative course of anti-TB drugs, all patients were cured of TB based on clinical response and serial chest radiographs. No morbidity related to pigtail drainage occurred.
Table 3 Change in morbidity visual analogue scale score (VAS) * after anti-tuberculous treatment with or without pigtail drainage

\begin{tabular}{lcc}
\hline & $\begin{array}{c}\text { Drainage group } \\
(\mathrm{n}=30)\end{array}$ & $\begin{array}{c}\text { No drainage } \\
\text { group }(\mathrm{n}=31)\end{array}$ \\
\hline Admission & $392.9(84.0)$ & $423.4(93.0)$ \\
1 week & $57.1(33.2)$ & $68.5(44.7)$ \\
2 weeks & $43.7(23.6)$ & $44.2(24.3)$ \\
4 weeks & $18.6(16.5)$ & $21.7(20.2)$ \\
8 weeks & $8.5(10.4)$ & $11.9(14.3)$ \\
12 weeks & $4.6(6.7)$ & $6.5(8.6)$ \\
24 weeks & $0 \quad(0)$ & $0 \quad(0)$ \\
\hline *Visual analogue scale (combined index score of \\
general well being, appetite, night sweating, \\
pleural chest pain, tiredness, dyspnoea, and \\
cough) at different evaluation time points. \\
Values are mean (SD).
\end{tabular}

\section{DISCUSSION}

Large et $^{4} \mathrm{l}^{4}$ have suggested a so-called "active" form of treatment of TB pleural effusions with repeated aspiration of the pleural cavity targeted to reduce the incidence of RPT. The positive effect of this treatment was noted before the routine use of rifampin. Dutt et $\mathrm{al}^{8}$ reported on the efficacy of a 6 month regimen of rifampin and isoniazid for tuberculous pleurisy in 1992, and this regimen has been widely used ever since to avoid repeated aspiration. Wyser et al reported a positive effect (improvement in symptom scores) following complete drainage in a prospective study of 70 patients with tuberculous pleural effusions. However, as these studies did not include controls, they were unable to determine whether early drainage was superior to no drainage over a long period of treatment.

Using a control group, our study has shown that the addition of pleural space drainage to anti-TB drug treatment had neither a beneficial effect on RPT development nor shortened the duration of fever or other clinical symptoms. The only positive effect found in the drainage group was a more rapid resolution of dyspnoea (median $4 v 8$ days between the two treatment groups). The incidence of RPT in all patients was about $50 \%$, which is similar to that found in previous studies. ${ }^{1-3}$ The incidence of RPT was similar whether or not pigtail drains were used. No significant difference in VAS score improvement was seen between the two groups after l week of treatment or during any scheduled follow up examination. Thus, while the combination treatment with anti-TB drugs appears key to improvement, the addition of pigtail drainage does not appear to make a significant difference.

RPT levels $>10 \mathrm{~mm}$ may have important clinical repercussions. In our study approximately $26 \%$ and $28 \%$ in the combined and single treatment groups, respectively, had RPT levels $>10 \mathrm{~mm}$. Similarly, Pablo et al $\mathrm{l}^{3}$ reported RPT levels of $>10 \mathrm{~mm}$ in about $19 \%$ of their patients with tuberculous pleurisy. Clinical and functional impairment induced by RPT is therefore not uncommon in these patients when treated with standard anti-TB drugs, whether or not the pleural effusion is drained.

Table 2 Outcome of pigtail drainage in patients with tuberculous pleurisy

\begin{tabular}{llll}
\hline & $\begin{array}{l}\text { Drainage group } \\
(n=30)\end{array}$ & $\begin{array}{l}\text { No drainage group } \\
(n=31)\end{array}$ & p value \\
\hline Median (IQR) days of fever & $0(0-6)$ & $0(0-5)$ & $0.769^{*}$ \\
Median (IQR) days of dyspnoea & $4(4-5)$ & $8(7-16)$ & $<0.001^{*}$ \\
Pleural thickening $>2 \mathrm{~mm}(\%)$ & $16(53)$ & $16(51)$ & $0.893 \dagger$ \\
Pleural thickening $>10 \mathrm{~mm}(\%)$ & $8(26)$ & $8(25)$ & $0.939 \dagger$ \\
Median (IQR) FVC \% predicted & $85.5(69-94)$ & $88(78-96)$ & $0.568^{*}$ \\
\hline IQR=interquartile range; FVC=forced vital capacity. & & \\
*Mann-Whitney U test. $\dagger \chi^{2}$ test & & \\
\end{tabular}


The results of our study indicate that, while RPT is clinically significant on initial presentation of tuberculous pleurisy, it may subside over time. Drainage of pleural effusions, even in the early stages (as in this study), does not prevent the development of RPT, but early diagnosis and early initiation of anti-TB drug treatment has been implicated in a decrease in the development of RPT in patients with tuberculous pleurisy. ${ }^{3}$

In conclusion, while early clearance of tuberculous pleural effusions by pigtail drainage improves dyspnoea, it does not appear significantly to decrease the incidence of RPT and other clinical symptoms following 1 week of treatment with anti-TB drugs. The development of RPT in itself may not be a serious clinical problem as it tends to subside over time, as long as the patient receives an adequate anti-TB treatment regimen.

Authors' affiliations

Y-F Lai, T-Y Chao, Y-H Wang, A-S Lin, Division of Pulmonary

Medicine, Department of Internal Medicine, Chang Gung Memorial

Hospital, Kaohsiung, Taiwan

\section{REFERENCES}

1 Barbas CS, Cukier A, Varvalho CR, et al. The relationship between pleural findings and the development of pleural thickening in patients with pleural tuberculosis. Chest 1991;100:1264-7.

2 Wyser C, Walzl G, Smedema JP, et al. Corticosteroids in the treatment of tuberculous pleurisy: a double-blind, placebo-controlled randomized study. Chest 1996;110:333-8.

3 Pablo A, Villena V, Echave-Sustaeta J, et al. Are pleural fluid parameters related to the development of residual pleural thickening in tuberculosis. Chest 1997; 112:1293-7.

4 Large SE, Levick RK. Aspiration in the treatment of primary tuberculous pleural effusion. BM 1958;1512-4.

5 Morrone N, Lombard, MC, Machado O. Prevention of pleural thickening through pleural aspiration in patients with tuberculous effusion. J Pneumol 1989;15:180-4.

6 American Thoracic Society. Snowbird workshop on standardization of spirometry. Am Rev Respir Dis 1979:119:831-8.

7 McCormoch HM, Horne DJ, Sheather S. Clinical applications of visual analogue scales: a critical review. Psychol Med 1988;18:1007-19.

8 Dutt AK, Moers D, Stead WW. Tuberculous pleural effusion. 6 months therapy with isoniazid and rifampin. Am Rev Respir Dis 1992;145: 1429-32.

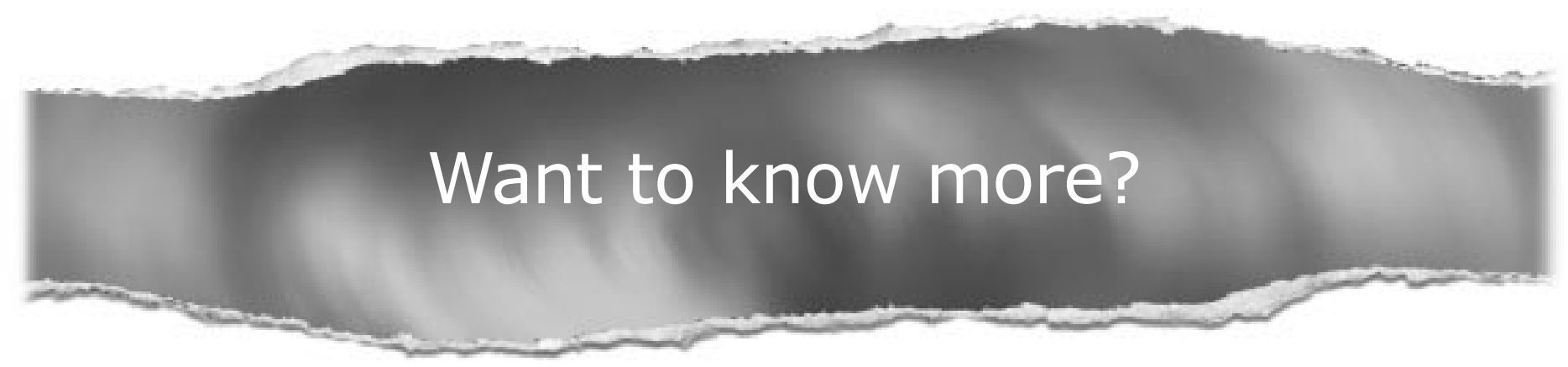

Data supplements

Limited space in printed journals means that interesting data and other material are often edited out of articles; however, limitless cyberspace means that we can include this information online. Look out for additional tables, references, illustrations.

www.thoraxjnl.com 